\title{
Jovian electrons in the inner heliosphere
}

\section{Proposing a new source spectrum based on $\mathbf{3 0}$ years of measurements}

\author{
A. $\operatorname{Vogt}^{1}$, B. Heber ${ }^{1}$, A. Kopp ${ }^{2,3}$, M. S. Potgieter ${ }^{3}$, and R. D. Strauss ${ }^{3}$ \\ ${ }^{1}$ Institut für Experimentelle und Angewandte Physik, Christian-Albrechts Universität zu Kiel, Leibnizstraße 11, 24118 Kiel, Germany \\ e-mail: vogt@physik.uni-kiel.de \\ ${ }^{2}$ Université Libre de Bruxelles, Service de Physique Statistique et des Plasmas, CP 231, 1050 Brussels, Belgium \\ ${ }^{3}$ Centre for Space Research, North-West University, 2520 Potchefstroom, South Africa
}

Received 8 August 2017 / Accepted 20 December 2017

\begin{abstract}
Context. Since the Pioneer 10 flyby of Jupiter it has become well known that electrons of Jovian origin dominate the lower MeV range of charged energetic particles in the inner heliosphere.

Aims. Because the Jovian source can be treated as point-like in numerical models, many attempts to investigate charged particle transport in the inner heliosphere have utilized Jovian electrons as test particles. The reliability of the derived parameters for convective and diffusive transport processes are therefore highly dependent on an accurate estimation of the Jovian source spectrum. In this study we aim to provide such an estimation .

Methods. In this study we have proposed a new electron source spectrum, specified at the boundary of the Jovian magnetosphere, fitted to flyby measurements by Pioneer 10 and Ulysses, with a spectral shape also in agreement with measurements at Earth's orbit by Ulysses, Voyager 1, ISEE and SOHO.

Results. The proposed spectrum is consistent with all previous theoretical suggestions, but deviates considerably in the lower MeV range which was inaccessible to those studies.
\end{abstract}

Key words. convection - astroparticle physics - Sun: heliosphere - interplanetary medium - methods: numerical - diffusion

\section{Introduction}

Cosmic rays (CRs), as charged particles, are continuously scattered by turbulent irregularities embedded in the heliospheric magnetic field. To correctly characterize the level of scattering, as quantified by the parallel and perpendicular diffusion coefficients (or, equivalently the corresponding mean-free-paths), still remains one of the biggest challenges in heliospheric physics. In the past, two different approaches have been followed. One approach is to specify the underlying level and structure of the turbulence and to calculate the diffusion coefficients by assuming some theoretical formulation (e.g., Engelbrecht \& Burger 2013). The other, more phenomenological and practical approach, is to determine and constrain the diffusion coefficients by comparing numerical model solutions with observations, thus establishing the spatial and rigidity dependence and the magnitude of the diffusion coefficients (e.g., Zhang et al. 2007).

A frequently used test case to determine transport parameters is the propagation of Jovian electrons, where the Jovian magnetosphere can the treated as a quasi-continuously emitting point-like source (see Pyle \& Simpson 1977). A first analytical model to study energetic particle propagation by means of Jovian electrons was introduced by Conlon (1978). Since then, various numerical models have been applied to study Jovian electron propagation in the inner heliosphere (see e.g., Fichtner et al. 2000; Ferreira et al. 2001; Strauss et al. 2011a, amongst others). A large uncertainty in these transport models is, however, the Jovian source function, that is, the magnitude and energy dependence of the Jovian electron distribution released from the Jovian magnetosphere. In order to produce satisfying and consistent model results, the source function must be constrained by in situ electron observations close to the Jovian magnetosphere. Previous attempts to construct a Jovian source function were at least partly based on measurements obtained at the Earth's orbit and therefore depend on transport effects. In this paper we propose a new Jovian source function which is based on spacecraft measurements obtained and published over the last 30 years. We will show that when temporal anomalies are ignored, the in situ observations of the source function by the Pioneer 10, Voyager 1 and Ulysses spacecraft are consistent with each other, while, when this new source function is introduced into a transport model, the resulting intensities are also consistent with various observations at the Earth's orbit.

\section{The Jovian source}

The first hint that Jupiter could serve as a dominant source of low-energy electrons in the inner heliosphere was given by McDonald et al. (1972) who pointed out that the characteristic $\sim 13$ month counting rate periodicities found in spacecraft data near Earth are in phase with Jupiter's synodical period. This hypothesis was confirmed by Teegarden et al. (1974), based on Pioneer 10 measurements, and since then by all spacecraft equipped with a particle detector that flew by Jupiter. The point-like nature of the Jovian source, at least on scales used for numerical simulation purposes was discovered by Pyle \& Simpson (1977), who disproved the earlier hypothesis that Jovian 
electrons are emitted through the planet's long magnetotail (see e.g., Pesses \& Goertz 1976). Furthermore they showed via the interaction of Jovian electrons and corotating interaction regions (CIRs) that each time Pioneer 10 was magnetically connected with the Jovian source the expected electron counting rate increase appeared, concluding that the source should be more or less continuous.

Regarding the question of how electrons could be accelerated up to several MeV within the Jovian magnetosphere, the evidence that these electrons originate in the solar wind and diffuse inward (see e.g., Bolton et al. 1989), suggests an acceleration via conservation of the first and second adiabatic invariant as shown by de Pater \& Goertz (1990). In situ observations by the Pioneer 11, Galileo and Cassini spacecraft (see van Allen et al. 1975; Fischer et al. 1996; Bolton et al. 2002) give a hint that additional processes such as wave particle interactions have to take place inside Jupiter's radiation belt, as discussed by Horne et al. (2008). Whether or not the findings of Simpson et al. (1992), indicating low-MeV electrons bursts with a quasi-periodicity of $\sim 40$ min throughout the dusk-side magnetosphere, are connected to these acceleration processes or even support theories of further acceleration outside the radiation belts, still needs to be clarified. Contributing to these issues, Juno measured $\mathrm{MeV}$ electrons in both the inner radiation belt and intense particle bursts at high latitudes outside the radiation belts (Becker et al. 2017), together with other observations of $\mathrm{MeV}$ and sub-MeV electrons in the Jovian polar region, which were detected while crossing magnetic field lines presumably mapping to the outer magnetosphere or even open field lines (see e.g., Clark et al. 2017; Zhang et al. 2017, amongst others). These findings therfore also revive the exploration of the release mechanism and contrast the findings of Galileo and Cassini which provided strong evidence that Jovian ions and electrons leak into interplanetary space despite a closed magnetic field topology, as stated by Krupp et al. (2002).

In recent years Cassini has also made corresponding observations within the Kronian magnetosphere, suggesting the acceleration of electrons up to relativistic and ultrarelativistic energies, as well as quasi-periodic one-hour pulsations in the outer magnetosphere according to Palmaerts et al. (2016) and Roussos et al. (2016), respectively. These findings add to the ongoing discussion whether there is a population of Kronian electrons in the inner heliossphere as stated by Lange \& Fichtner (2008). An improved understanding of the Jovian source and the corresponding electron spectrum would therefore also help to distinguish between Galactic, Jovian and a possible weaker Kronian electron population.

\section{Electron measurements in the inner heliosphere}

A major challenge for any attempt to study Jovian electrons is the limited amount of in situ observations of low energy electrons near the Jovian magnetosphere. For this reason, this study combines electron spectra obtained by several spacecraft missions since 1973, both at the Earth's orbit and near to the Jovian magnetosphere. Thereby the flyby spectra were used to derive our proposed source spectrum, while the Earth orbit spectra were taken into account to check the consistency. These observations as listed in Table 1 are briefly discussed below.

\subsection{Flyby observations}

Figure 1 shows energy spectra obtained outside of Jupiter's magnetosphere during the Pioneer 10 and Ulysses flybys (for

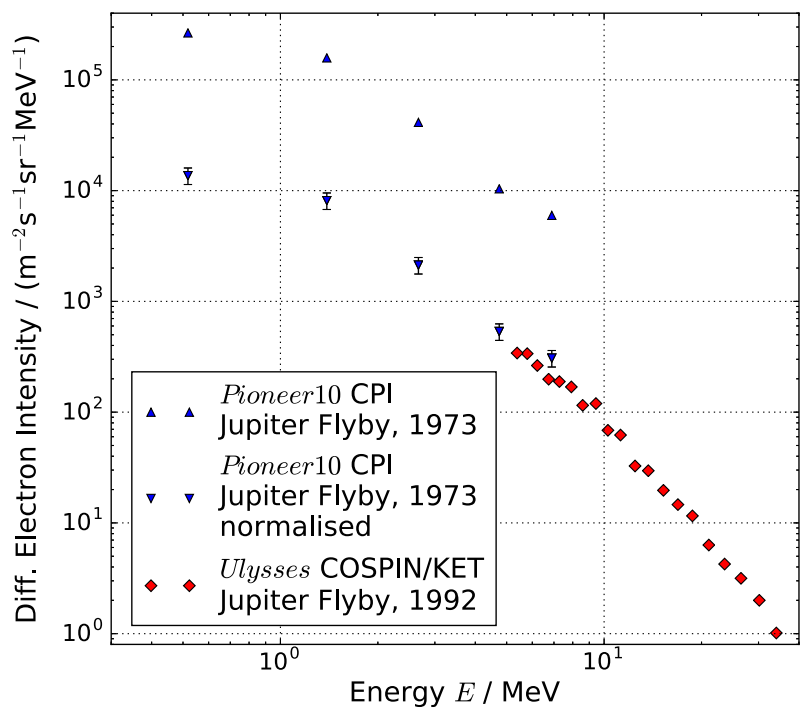

Fig. 1. Electron energy spectra measured by Pioneer 10 and Ulysses KET. The Pioneer 10 spectrum is shown both with and without the renormalization illustrated in Fig. 2 and described in Sect. 3.1.

details, see Table 1). The COsmic and Solar Particle INvestigation Kiel Electron Telescope (COSPIN-KET) on board Ulysses measured electron counting rates in three energy channels during the entire mission from October 1990 until June 2009. Using a detailed data analysis utilizing GEANT 3 simulations Heber et al. (2005) could provide two electron spectra: one measured in the early cruise phase still near Earth's orbit and a second one capturing the electron fluxes during the first Jupiter encounter in February 1992. Both COSPIN-KET spectra are consistent with spectra obtained by the Voyager 1 BSe detector, for equivalent energies both at the Earth and for the Jupiter encounter in 1977. These observations were made available to Nndanganeni (2015), through private communication between W. R. Webber and M. S. Potgieter, but are otherwise not yet published. Therefore, the BSe spectra were only taken into account for comparison to and evaluation of COSPIN-KET data.

While the COSPIN-KET and BSe data are found to be consistent with each other, the Pioneer 10 spectrum differs by a factor of more than one order of magnitude. Teegarden et al. (1974) determined their energy spectrum from measurements obtained on 11 May 1973, a day with extraordinary high fluxes. Due to statistical measures, this spectrum as shown in Fig. 1 and another one based on measurements inside the outer Jovian magnetosphere, were the only electron spectra published by the Pioneer 10 CPI team. No spectra covering the quiet times while Pioneer 10 was approaching and/or leaving Jupiter are available. The reason for this observation of high electron fluxes which made it possible to obtain a statistically trustworthy spectrum, lies in the occurrence of a so-called Jovian electron jet, a phenomena discovered first in the data of the Ulysses spacecraft (Ferrando et al. 1993) and later also during the Pioneer 10 period (Dunzlaff et al. 2009): such electron events can be identified as Jovian jets by three matching criteria. A short-duration increase in the electron flux is observed together with a significant anisotropy and a local interplanetary magnetic field aligned to the direction toward Jupiter. Furthermore, the spectral slope is the same as the one observed inside the Jovian magnetosphere. Due to this same spectral slope despite varying intensities, the spectral slope of Jovian jets is also believed 
Table 1. Overview of the electron spectra used in this study.

\begin{tabular}{lll}
\hline \hline Location of observation & Spacecraft mission/instrument & Source \\
\hline Flyby spectra & Pioneer 10 Charged Particle Instrument (CPI) & Teegarden et al. (1974) \\
& Ulysses Kiel Electron Telescope (KET) & Heber et al. (2005) \\
\hline \multirow{2}{*}{ Earth orbit spectra } & SOHO/EPHIN & Kühl et al. (2013) \\
& Ulysses Kiel Electron Telescope (KET) & Heber et al. (2005) \\
& ISEE 3 (ICE) Chicago electron spectrometer & Moses (1987) \\
\hline
\end{tabular}

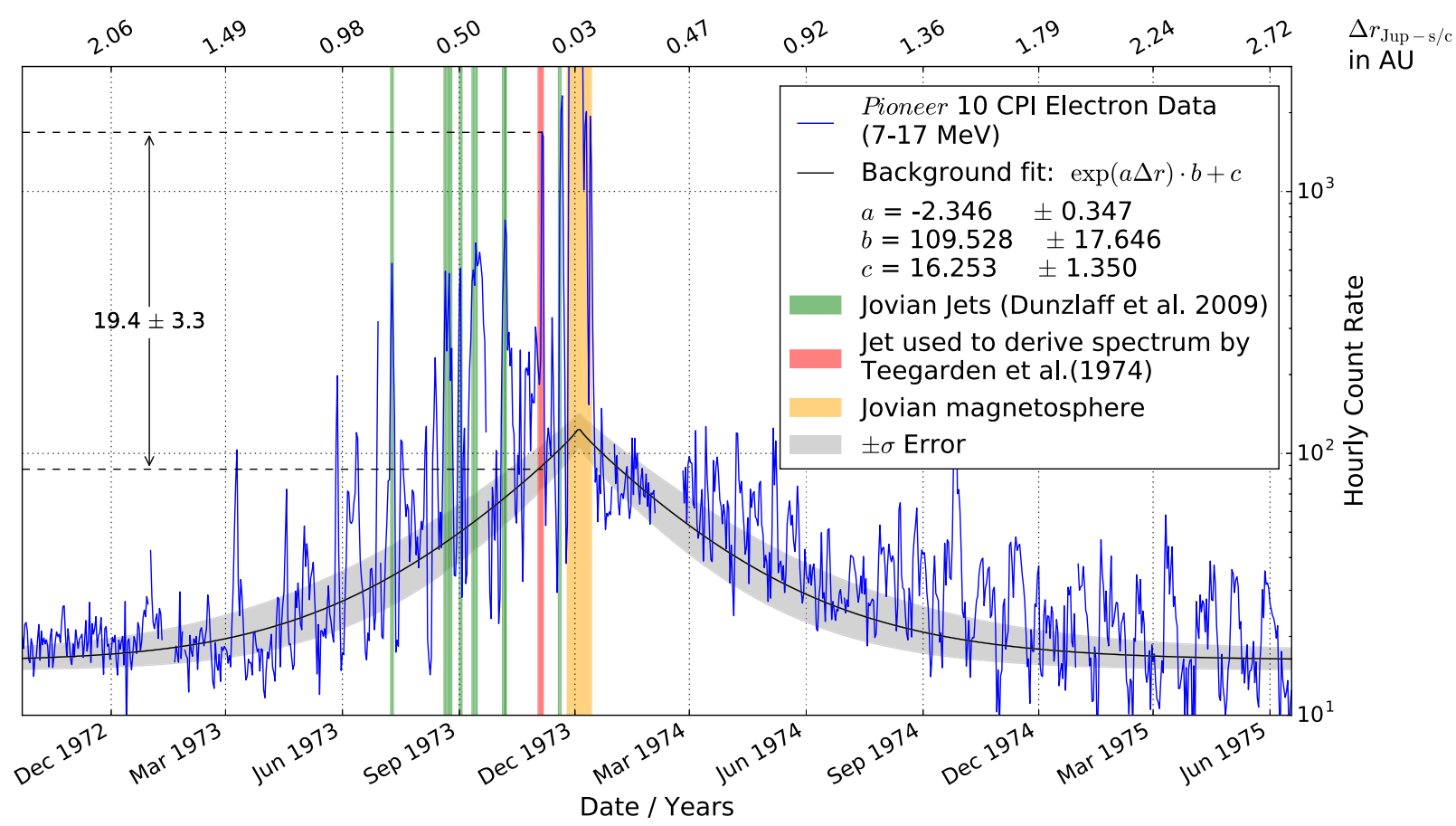

Fig. 2. Pioneer 10's CPI counting rates of the $7-17 \mathrm{MeV}$ electron channel (blue), are displayed together with the result of an exponential background fit (black with a gray shaded region showing a $1 \sigma$ uncertainty). In order to estimate the increase of the electron flux in the Jovian jet used to determine the Pioneer spectrum by Teegarden et al. (1974; shaded in red) all other jets identified by Dunzlaff et al. (2009; green) were also excluded from the background, along with the fluxes obtained inside the Jovian magnetosphere (orange).

to be similar to the quiet time Jovian population's without significant effects of the possibly different release mechanisms. This assumption of Ferrando et al. (1993) is supported by the agreement with the COSPIN-KET data in the energy range covered by both spacecraft, as well as by our check for consistency with Earth-orbit observations in Sect. 5. Because the electron event, which Teegarden et al. (1974) used to obtain their source spectrum, is actually included in the list of electron jets by Dunzlaff et al. (2009), it can be normalized to the quiet-time background flux to be used in a meaningful manner.

Figure 2 shows the daily Pioneer 10 electron fluxes measured by the Charged Particle Instrument $(\mathrm{CPI})^{1}$ together with the result of an exponential background fit (black with a gray shaded region showing a $1 \sigma$ uncerntainty). In order to estimate the increase of the electron flux in the Jovian jet used to determine the Pioneer spectrum by Teegarden et al. (1974) all other jets identified by Dunzlaff et al. (2009) were also excluded from the background, along with the fluxes obtained inside the Jovian magnetosphere. The comparison between the measured

\footnotetext{
1 Taken from: ftp://spdf.gsfc.nasa.gov/pub/data/pioneer/ pioneer10/particle/cpi/ip_1day_ascii/p10cpi.h24
}

flux and the fitted background gives a hint that the intensity of the Pioneer 10 spectrum has to be renormalized and reduced by a factor of about 20, which agrees quite well with the Ulysses COSPIN-KET spectrum as shown in the lower panel of Fig. 1. The $\pm \sigma$ uncertainty was computed by multiplying the Jacobian matrix with the residual variances, estimated by the mean square errors, according to the documentation of the fitting routine ${ }^{2}$. The resulting covariance matrix has been used to derive the standard error and therefore the $\pm \sigma$ uncertainty. Applying this renormalization factor to the Teegarden et al. (1974) data leads to a convincing agreement between the Pioneer 10, Ulysses and Voyager data (not shown here) as displayed in Fig. 1.These consistent observations can now be used to derive a Jovian source function, as it is shown by the fit in Fig. 3. Because of their spatial and temporal constraints, Jovian jets are not likely to influence the strength of the Jovian source. Temporal varitions due to the passing of a jet are only to expected inside a radius of $\sim 0.8 \mathrm{AU}$ around Jupiter, further inside the interplanetary space jets have not been detected yet.

\footnotetext{
2 Provided at https://docs.scipy.org/doc/scipy-0.19.0/ reference/generated/scipy.optimize. leastsq.html
} 
Although three other spacecraft visited the Jovian magnetosphere in recent years, namely Galileo, Cassini and Juno, no flyby spectra suitable for this study have been published yet. Considering the instrumentation of all three missions, the energetic particle detector (EPD) onboard Galileo (see Williams et al. 1992), the Low Energy Magnetospheric Measurement System (LEMMS) onboard Cassini (see Krimigis et al. 2004; Haggerty \& Livi 2004) as well as Juno's Jupiter Energetic particle Detector Instrument (JEDI) discussed by Mauk et al. (2013), the energy range of interest seems to be well covered. Observations inside the Jovian and Kronian magnetosphere, respectively, as briefly discussed in Sect. 2 suggest also the possibility of deriving such electron spectra close to but not inside the Jovian magnetosphere. Regarding the well known difficulties obtaining such spectra due to the complexity of the corresponding response function, as detailed in Heber et al. (2005) and Kühl et al. (2013), amongst others, deriving these spectra from the published counting rates is beyond the scope of this study. Nevertheless any flyby spectrum of Galileo, Cassini or Juno would contribute to constrain the Jovian source spectrum further in this energy range.

\subsection{Observations at Earth's orbit}

Although commonly used in studies on this subject, electron spectra at the Earth's orbit will not be used in this study to obtain a Jovian source spectrum. The more detailed measurements at $1 \mathrm{AU}$ are however important in order to validate our findings.

In addition to the already mentioned Ulysses COSPINKET and Voyager 1 BSe data, the electron spectral data of SOHO-SEPT cover roughly the same energy range in more detail as the flyby data by Pioneer 10. As described by Kühl et al. (2013) two electron spectra were derived from SOHO-EPHIN (Müller-Mellin et al. 1995) data to investigate the effect of parallel and perpendicular diffusive transport on Jovian electron fluxes. In our work, we chose the spectrum which corresponds to time periods of undisturbed magnetospheric conditions between Jupiter and the spacecraft, as well as an (assumed) magnetic connection. Therefore, it is reasonable to neglect perpendicular diffusion in order to compare the spectrum to the flyby spectra in the relevant energy range. This reduces the probability that other effects, apart from adiabatic deceleration (energy loses), influence the spectral shape and intensity.

The same decision to take only data of best magnetic connectivity into account was made for the electron spectra derived by Moses (1987) from the data obtained by the Chicago electron spectrometer on board ISEE 3 (ICE). The data set used for our validation in Sect. 5 consists of six energy spectra obtained between 1978 and 1984 in time intervals with a period (length) up to 200 days when ISEE 3 was believed to be magnetically connected with the Jovian source

- 1978 November 4 to 1979 April 10;

- 1979 September 7 to 1980 April 9;

- 1980 October 26 to 1981 April 20;

- 1981 October 27 to 1982 June 19;

- 1982 November 28 to 1983 may 20;

- 1983 December 6 to 1984 July 18

The variations in the different energy channels are due to the long averaging periods in connection with the variability of both the connectivity and the solar activity during that time, although flares identified by IMP 8 were excluded. In contrast to previous studies on Jovian electrons observed at Earth, the data product derived from these six previously discussed spectra (which was an assumption of the source itself) was disregarded in favor of

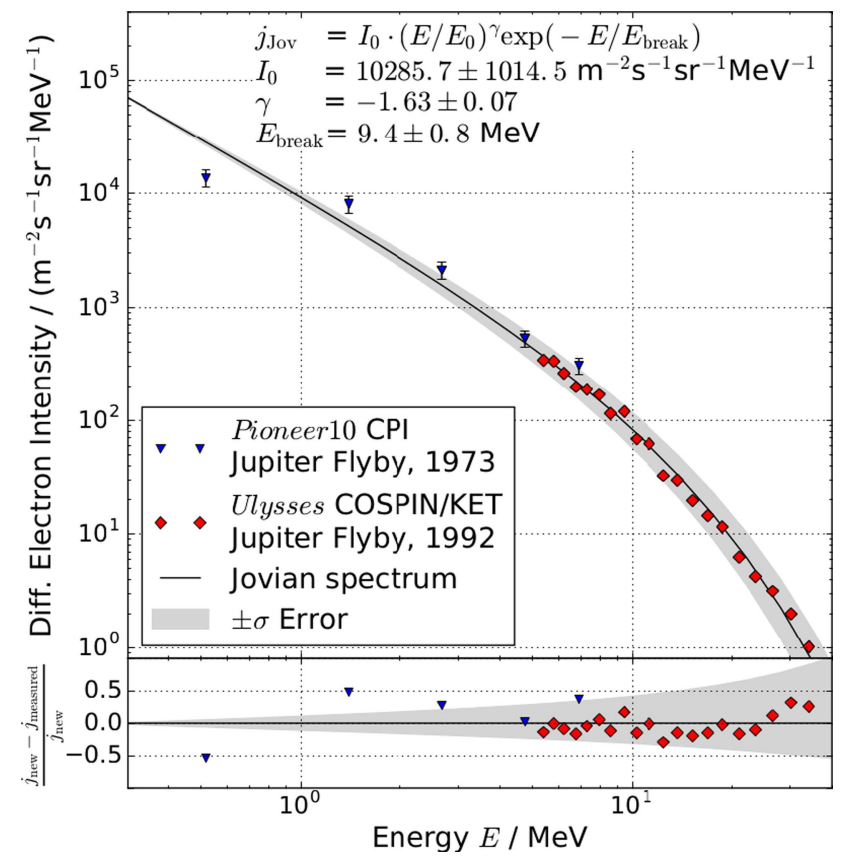

Fig. 3. Proposed new Jovian spectrum according to Eq. (11) together with the Pioneer $10 \mathrm{CPI}$ and Ulysses COSPIN-KET data. The $\pm \sigma$ uncertainty has been derived by the same method as detailed in Fig. 2.

the original data as shown in Fig. 8. of Moses (1987). We will revisit this matter in Sect. 5 when discussing previous attempts to estimate the Jovian source spectrum.

\section{The new source spectrum}

A first attempt to estimate a Jovian source spectrum was made by Teegarden et al. (1974) who suggested a power law with an exponent of about -1.5 , in terms of kinetic energy, based on their own Pioneer 10 data, as shown in Fig. 1, and the findings of McDonald et al. (1972) who analyzed electron spectra at the Earth's orbit. This first, mostly qualitative suggestion

$j(E) \propto\left(\frac{E}{E_{0}}\right)^{-1.5}$,

normalized to $E_{0}=1 \mathrm{MeV}$, was expanded by Baker \& van Allen (1976) by adding a second power law for the steepening of the spectrum at higher energies. These two approaches were elaborated on as more data became available. In the following we present a selection of the most significant suggestions based mainly on these two assumptions before discussing the reasons that lead us to propose a new Jovian spectrum with a different mathematical expression.

Following the initial approach by Teegarden et al. (1974), Eraker (1982) presented a first quantitative analysis of the Jovian source and concluded the source function as

$J(E)=310^{25}\left(\frac{E}{E_{0}}\right)^{-1.7}$,

in units of electrons $\mathrm{s}^{-1} \mathrm{MeV}^{-1}$, taking into account the data of Pioneer 10, IMP 8 and Mariner 10 as well as the results of the first Jovian electron propagation model developed by Conlon (1978). This work was continued by Lopate (1991), who investigated the spectral index during the whole Pioneer 10 mission up 
to 1991 and found that the ratio between the 27-day averages of 2-7 MeV and 7-17 MeV electrons was quite stable during the flight, and showed only minor fluctuations with a slight decrease during the 1987 solar minimum period when the spacecraft was already at a radial distance of $40 \mathrm{AU}$.

Also relying on Pioneer 10 and IMP 8, but regarding Pioneer 8/9 data as well, Haasbroek et al. (1997) specified the approach of Baker \& van Allen (1976) quantitatively and concluded

$j(E)=c\left(\frac{E}{E_{0}}\right)^{-1.5}\left(1+\frac{E}{5 E_{0}}\right)^{-3.5}$,

again with $E_{0}=1 \mathrm{MeV}$ and in units of electrons $\mathrm{m}^{-2} \mathrm{~s}^{-1} \mathrm{sr}^{-1} \mathrm{MeV}^{-1}$ to simulate the Jovian electron contribution on electron counting rates throughout the heliosphere. The normalization factor $c$ was obtained via the Jovian electron data of Pioneer 10 and ISEE 3 and given indirectly by referring to corresponding measurements. Reconstruction this normalization suggest that $c=4 \cdot 10^{4}$, the value which was used to compare the spectrum in Fig. 4, provides the least deviation to the data the spectrum is based on.

Also, based on the spectral shape concluded according to the ISEE 3 data by Moses (1987) and the flyby data of the Pioneer 10 mission, Ferreira et al. (2001) expanded this approach of utilizing a soft and a hard power law to describe the Jovian source function, as displayed in Fig. 4 by the orange line. In addition to the first power law which is in agreement with the previous suggestions

$j(E)_{1.5}=5 \cdot 10^{3}\left(\frac{E}{E_{0}}\right)^{-1.5}$,

with

$E_{0}=1 \mathrm{GeV}$,

the second power law added to cover the steepening at higher energies was much harder than the earlier approach by Haasbroek et al. (1997):

$j(E)_{6.0}=10^{9}\left(\frac{E}{E_{0}}\right)^{-6.0}$

matching the normalized ISEE 3 (ICE) spectral data by Moses (1987), mentioned in Sect. 3.2. These two power laws are combined in a spectral function as

$j(E)_{\text {Jovian }}=1.5\left(\frac{c_{k} \cdot j_{1.5} \cdot d_{k} \cdot j_{6.0}}{c_{k} \cdot j_{1.5}+d_{k} \cdot j_{6.0}}\right)$,

again in units of electrons $\mathrm{m}^{-2} \mathrm{~s}^{-1} \mathrm{sr}^{-1} \mathrm{MeV}^{-1}$ and with the fitting parameters

$c_{k}=0.6$,

and

$d_{k}=5.0$.

The Jovian source spectrum by Ferreira et al. (2001) was used in several numerical modeling studies in the subsequent years, covering many aspects and issues of charged particle physics in the inner heliosphere. See for example, Ferreira \& Potgieter (2002); Ferreira et al. (2003) and the review by Ferreira (2005).

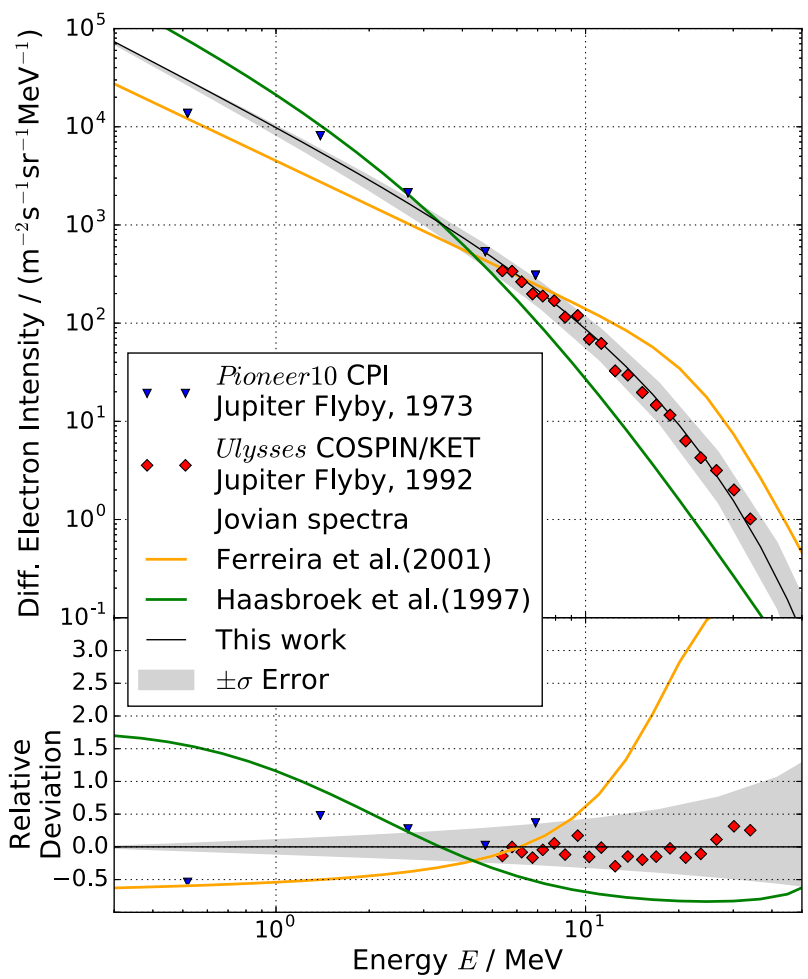

Fig. 4. New source spectrum alongside that by Ferreira et al. (2001) in orange and the suggestion by Haasbroek et al. (1997; green) as discussed in Sect. 4. In the upper panel all three spectra are shown alongside the Jovian flyby data and the $1 \sigma$ error region surrounding our estimation. The lower panel shows the relative deviation of the estimations, calculated as $\left(j_{\text {previous }}-j_{\text {new }}\right) / j_{\text {new }}$. Especially in the energy range above $E_{\text {break }}=9.4 \mathrm{MeV}$ the lower panel shows how the deviation increases up to over $100 \%$ and even far more for the spectrum by Ferreira et al. (2001).

However, with more and more electron data made available, it has become an important and relevant task to revisit the question whether the source function could be simply updated or if it would be possible and necessary to establish a new one. A main scientific goal to understand cosmic ray modulation and transport has been to establish a local interstellar spectrum (LIS) and compare its shape with the corresponding particle spectrum measured in the inner heliosphere. In order to do so, it is necessary to distinguish between the Jovian and the Galactic components but with new computations of the electron LIS by Potgieter \& Nndanganeni (2013), Potgieter et al. (2015), Bisschoff \& Potgieter (2014) and Potgieter \& Vos (2017), see also the overview by Potgieter (2014), based on measurements by Voyager 1 in the very local interstellar medium, the suspicion raised that the Jovian source spectrum overestimated the amount of electrons in the low-MeV range; see for example, the discussion by Nndanganeni \& Potgieter (2016). Also the data from the Ulysses flyby and the knowledge of the Voyager BSe spectral data suggested that the spectrum had to be corrected to cover the spectral shape in that same energy range correctly.

Therefore, the decision was made to combine the most significant measurements of electron spectra in the Jovian electron energy range, both at Jupiter and at the Earth's orbit, as listed in Table 1 and as discussed before. In order to make our result self consistent the flyby spectra were used to obtain the spectral shape and the quantitative fit, while the Earth orbit data provided the possibility of a fully independent check for consistency check at $1 \mathrm{AU}$. 
Regarding the general shape we follow neither of the introduced approaches but the combination of a power-law with an exponential cut-off

$j(E)_{\text {jov }} \propto\left(\frac{E}{E_{0}}\right)^{\gamma} \mathrm{e}^{-E / E_{\text {break }}}$,

as particle acceleration theory suggests; see for example, the discussion of diffusive shock acceleration by Prinsloo et al. (2017).

This approach, again normalized with $E_{0}=1 \mathrm{MeV}$ was fitted via the least-square method taking only the flyby data of Pioneer 10 CPI and Ulysses COSPIN-KET into account. The result is displayed in Fig. 3 together with the detailed uncertainties of the fit and in Fig. 4 compared with the estimates of Eqs. (3) and (7) and has the following mathematical expression for the Jovian source function:

$j(E)_{\text {jov }}=1.029 \cdot 10^{4}\left(\frac{E}{E_{0}}\right)^{-1.63} \mathrm{e}^{-E / E_{\text {break }}}$,

with

$E_{\text {break }}=9.4 \mathrm{MeV}$.

\section{Discussion}

In order to check for consistency, we simulated the resulting electron fluxes at the Earth orbit for times with the best magnetic connection and compared them to the corresponding spacecraft data as listed in Table 1. The result is shown in Fig. 5 together with the source spectrum and the flyby data. For our simulation we used the stochastic differential equation method as described in detail by Kopp et al. (2012). The numerical model by Dunzlaff et al. (2015), used in this study, implements the parallel mean free path

$\lambda_{\|}(r)=\frac{\lambda_{0}}{2}\left(1+\frac{r}{r_{0}}\right)$,

depending on a mean free path $\lambda_{0}$ at a point of reference $r_{0}$, which is in this case the Earth orbit at $1 \mathrm{AU}$. The parallel mean free and the particle's speed $v$ then scale the parallel diffusion coefficient as

$\kappa_{\|}(r)=\frac{v \lambda_{\|}(r)}{3}$,

and the perpendicular diffusion coefficient via the proportional factor $\chi$

$\kappa_{\perp}(r)=\chi \kappa_{\|}(r)$.

As input parameters we choose a constant value for the solar wind speed of $u_{S W}=400 \mathrm{~km} \mathrm{~S}^{-1}$ and a ratio between the parallel and perpendicular diffusion coefficient of $\kappa_{\|} / \kappa_{\perp}=0.02$, The value for the parallel mean free path at $1 \mathrm{AU}$ was set to $\lambda_{0}=0.1 \mathrm{AU}$, as suggested theoretically by Palmer (1982) and implemented successfully by Strauss et al. (2011b) in a comparable model setup. Despite the necessity that future work has to further investigate the chosen transport parameters with a more elaborated approach, the simulated electron spectrum at the Earth's orbit confirms that the proposed source spectrum can reproduce the observed data.

Comparing our proposed expression for the Jovian source function qualitatively with the previous suggestions, as shown in

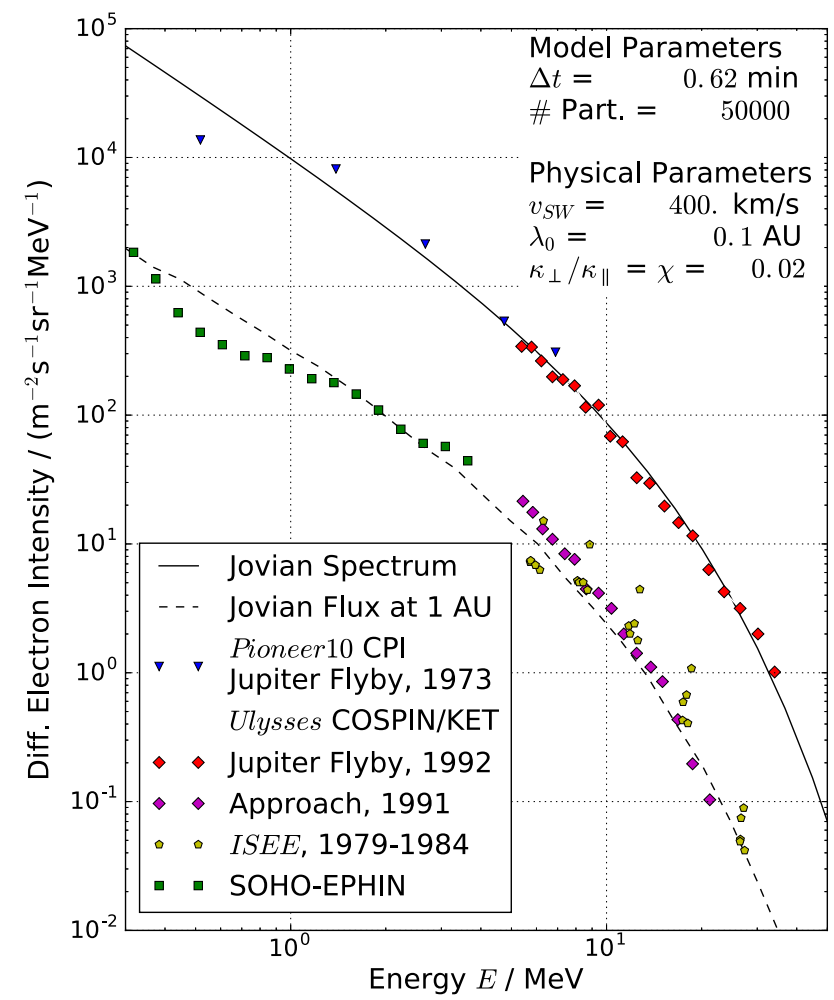

Fig. 5. New Jovian source spectrum (solid line) and the corresponding simulated intensities at the Earth's orbit (dashed line) at times of good magnetic connectivity to the source. Additional to the flyby spectra obtained by Pioneer $10 \mathrm{CPI}$ and Ulysses COSPIN-KET which were introduced to derive the source, here also the spectral data by SOHOEPHIN (green), ISEE 3 (yellow), and the Ulysses COSPIN-KET (violet) data measured shortly after the launch are displayed, as documented in Table 1. While the SOHO-EPHIN spectrum is sampled over several time periods of good magnetic connectivity with the source, due to reasons explained in Sect. 3.2 the spectra obtained by ISEE 3 have to be included separately.

Fig. 4, our value for the exponent $\gamma=-1.63$ ranges in between the values given in Eq. (2) by Eraker (1982) and Eq. (3) by Haasbroek et al. (1997) or Eq. (7) by Ferreira et al. (2001), respectively. In contrast to the approach in Eq. (7), where the softening of the spectrum is treated by an additional power with exponents of -3.5 or -6 in the second case, applying an exponential cut-off creates a much smoother transition between the harder and softer ranges of the spectrum. The even smoother spectral shape given by Haasbroek et al. (1997) in Eq. (3) seems to be too steep in the lower energy range and to flat in the higher energy range.

Furthermore the value of $\gamma=-1.63$ for the exponent in our findings contribute to the discussion about a common acceleration process throughout the heliosphere as introduced by Fisk \& Gloeckler (2014). By that means the newly established source function provides a benchmark to study and explain electron acceleration, being more consistent with contemporary theory, if one would not assume two equally effective acceleration processes within the Jovian magnetosphere to create the spectral overlap.

The quantitative comparison with the older estimates, especially with the spectrum proposed by Ferreira et al. (2001) in Fig. 4, shows that the new source function gives higher electron intensities in the lower energy range of the spectrum, up to one or two MeV. This can easily be explained by the fact that up to our renormalization of the Pioneer 10 CPI data no reliable 
flyby measurements were available in that spectral range. Also, the SOHO-EPHIN data at the corresponding energy range show an unexplained spectral break at $0.5 \mathrm{MeV}$, which either could be caused by instrumental effects or even a different energy dependence of the electron mean free path in this energy range, as stated by Kühl et al. (2013). To avoid assumptions which are not clearly supported by statistical evidence, the functional form was limited to the chosen approach. Due to the poor data coverage at the Jovian magnetosphere and the uncertainties of the Earth orbit data, the $\mathrm{keV}$ and low $\mathrm{MeV}$ range therefore has to be treated carefully, until further flyby measurements become available, as already discussed in Sect. 3.1. Despite these concerns, the convincing consistency check and the fact, that the break energy $E_{\text {break }}=9.4 \mathrm{MeV}$ is located in an well covered energy range, both suggest that only minor changes are to be expected regarding the intensity or the exponent $\gamma=-1.63$.

Therefore, in the spectral range above $4-5 \mathrm{MeV}$, we expect lower fluxes than previously thought, as supported by the Ulysses COSPIN-KET flyby data, which also were not available at the times the older estimates were published. In this energy range, especially of 30-50 MeV, the second power law of the Ferreira et al. (2001) approach would give an overestimation of electrons up to $60 \%$ and even more. In particular, the second panel of Fig. 4 illustrates these differences, caused by the different data sets used to constrain the spectra. As briefly mentioned in Sect. 3.2 we did not consider the final data product of Moses (1987), on which Ferreira et al. (2001) based their assumption in the low $\mathrm{MeV}$ range, in favor of the uncorrected spectra. The deviation of this finally published spectrum to the corresponding data obtained by Ulysses COSPIN-KET and Voyager $1 \mathrm{BSe}$ becomes reasonable considering that and how Moses (1987) estimated the Jovian source spectrum. The Moses (1987) data was corrected in both absolute intensity and spectral shape, due to the estimation of a dominant solar component and the results of Monte Carlo transport simulations with a strong influence of adiabatic deceleration. An inspection of current approximations of adiabatic energy changes, following the suggestion of Fichtner et al. (2000), indicate that significant changes in the spectral shape are expected at much higher energies, casting doubt on this approach. Furthermore, with the spectral data of Ulysses COSPIN-KET and Voyager $1 \mathrm{BSe}$, flyby measurements became available, which are by definition independent of any assumptions on particle transport.

Taking into account that the most prominent deviations from previous works, both in shape and intensity, are located in the $\mathrm{MeV}$ range suggests to revisit past simulations concerning Jovian electrons, because mostly $\mathrm{MeV}$ electron counting rates were used for evaluation. Normalizing the simulation results by an overestimated source intensity thereby would have lead to correspondingly higher fluxes. Concluding that past simulations which seemed to match the measured counting rates could have actually produced fluxes lower than observed, one would expect that the transport parameters have to be changed in order to allow a more efficient transport to reproduce the measurements.

Another consequence could be that estimates on the ratio between the Galactic and the Jovian contribution to electron fluxes at the Earth (see e.g., Strauss et al. 2013) would have to be reconsidered based on the effects the new source function has on the computer modeled Jovian electron intensities.

\section{Conclusion}

In contrast to previous attempts the newly developed Jovian source function as formulated in Eq. (11) is derived only from flyby data without taking Earth orbit measurements into account. Because our new source spectrum is essentially independent on any assumptions regarding charged particle transport, Jovian electron simulations based on our source spectrum can be considered more self-consistent than was possible before. The consistency check shown in Fig. 5 suggests that more elaborated model set-ups and parameter studies can reproduce the measurements at Earth orbit as listed for the spectral data in Table 1 with more accuracy and reliability.

Looking at the theoretical implications of our findings, the new source spectrum agrees with the assumptions about charged particle acceleration theory. Therefore we could provide an orientation for investigations focused on the acceleration and transport processes inside the Jovian magnetosphere (as referred to briefly in Sect. 2) to constrain their models to a comparable spectral slope and intensity outside the magnetosphere or inside a Jovian jet.

Another possible outcome for related research fields is to address the efficiency of perpendicular diffusion with respect to field line random walk. Because the continuous outflow is assumed as perpendicular diffusion through a closed field line topology (see Krupp et al. 2002), comparing the background of Jovian electrons to the peak intensities during Jovian jets which are magnetically connected to the Jovian magnetosphere would provide an estimate.

Most importantly, the new Jovian source spectrum established in this work allows future studies to investigate charged particle transport and all it's intricate effects with much improved reliability. In particular, the ratio of Galactic to Jovian electron intensities in the inner heliosphere below about $50 \mathrm{MeV}$ can now be done more convincingly so that a better estimate of how many low-energy Galactic cosmic ray electrons actually reach the Earth is possible, as discussed in Sect. 5. Also the influence of the latitudinal transition between fast and slow solar wind and the different propagation conditions during solar minima and maxima could be revisited. By continuing the investigations of investigations as done by Moeketsi et al. (2005), modeling the low $\mathrm{MeV}$ electron counting rates obtained by Ulysses COSPINKET addresses both the efficiency of perpendicular diffusion up to the polar regions and the effects of the changing solar wind speed on particle transport.

Regarding the influence of the source function, not only on computed intensities, but also on the estimated propagation time as shown in Vogt et al. (2015), it seems promising that after more than 30 years of measurements the investigation of Jovian electron transport can still lead to new insights on the propagation of low-energy electron in the heliosphere.

Acknowledgements. The work was partly carried out within the framework of the bilateral BMBF-NRF-project "Astrohel" (01DG15009) as supported by the Bundesministerium für Bildung und Forschung (BMBF) and the South African National Research Foundation (NRF), grant UID93132.

\section{References}

Baker, D. N., \& van Allen, J. A. 1976, J. Geophys. Res., 81, 617

Becker, H. N., Santos-Costa, D., Jørgensen, J. L., et al. 2017, Geophys. Res. Lett., 44, 4481

Bisschoff, D., \& Potgieter, M. S. 2014, ApJ, 794, 166

Bolton, S. J., Gulkis, S., Klein, M. J., de Pater, I., \& Thompson, T. J. 1989, J. Geophys. Res., 94, 121

Bolton, S. J., Janssen, M., Thorne, R., et al. 2002, Nature, 415, 987

Clark, G., Mauk, B. H., Paranicas, C., et al. 2017, Geophys. Res. Lett., 44, 4419

Conlon, T. F. 1978, J. Geophys. Res., 83, 541

de Pater, I., \& Goertz, C. K. 1990, J. Geophys. Res., 95, 39 
Dunzlaff, P., Kopp, A., Heber, B., \& Sternal, O. 2009, Proc. 31st Int. Cosmic Ray Conf.

Dunzlaff, P., Strauss, R. D., \& Potgieter, M. S. 2015, Comput. Phys. Commun., 192,156

Engelbrecht, N. E., \& Burger, R. A. 2013, ApJ, 772, 46

Eraker, J. H. 1982, ApJ, 257, 862

Ferrando, P., Ducros, R., Rastoin, C., \& Raviart, A. 1993, Planet. Space Sci., 41, 839

Ferreira, S. E. S. 2005, Adv. Space Res., 35, 586

Ferreira, S. E. S., \& Potgieter, M. S. 2002, J. Geophys. Res. (Space Phys.), 107, 1221

Ferreira, S. E. S., Potgieter, M. S., Burger, R. A., Heber, B., \& Fichtner, H. 2001 J. Geophys. Res., 106, 24979

Ferreira, S. E. S., Potgieter, M. S., Moeketsi, D. M., Heber, B., \& Fichtner, H 2003, ApJ, 594, 552

Fichtner, H., Potgieter, M., Ferreira, S., \& Burger, A. 2000, Geophys. Res. Lett., 27, 1611

Fischer, H. M., Pehlke, E., Wibberenz, G., Lanzerotti, L. J., \& Mihalov, J. D. 1996, Science, 272, 856

Fisk, L. A., \& Gloeckler, G. 2014, J. Geophys. Res. (Space Phys.), 119, 8733

Haasbroek, L. J., Potgieter, M. S., \& Le Roux, J. A. 1997, Adv. Space Res., 19, 953

Haggerty, D. K., \& Livi, S. 2004, Adv. Space Res., 33, 2303

Heber, B., Kopp, A., Fichtner, H., \& Ferreira, S. E. S. 2005, Adv. Space Res., 35, 605

Horne, R. B., Thorne, R. M., Glauert, S. A., et al. 2008, Nat. Phys., 4, 301

Kopp, A., Büsching, I., Strauss, R. D., \& Potgieter, M. S. 2012, Comput. Phys. Commun., 183, 530

Krimigis, S. M., Mitchell, D. G., Hamilton, D. C., et al. 2004, Space Sci. Rev., 114,233

Krupp, N., Woch, J., Lagg, A., et al. 2002, Geophys. Res. Lett., 29, 1736

Kühl, P., Dresing, N., Dunzlaff, P., et al. 2013, Proc. 33rd Int. Cosmic Ray Conf.

Lange, D., \& Fichtner, H. 2008, A\&A, 482, 973

Lopate, C. 1991, Proc. 22th Int. Cosmic Ray Conf.
Mauk, B. H., Haggerty, D. K., Jaskulek, S. E., et al. 2013, Space Sci. Rev. McDonald, F. B., Cline, T. L., \& Simnett, G. M. 1972, J. Geophys. Res., 77, 2213 Moeketsi, D. M., Potgieter, M. S., Ferreira, S. E. S., et al. 2005, Adv. Space Res., 35, 597

Moses, D. 1987, Astrophys. J., Part 1, 313, 471

Müller-Mellin, R., Kunow, H., Fleißner, V., et al. 1995, Solar Phys., 162, 483

Nndanganeni, R. R. 2015, PhD thesis, North-West University, South Africa

Nndanganeni, R. R., \& Potgieter, M. S. 2016, Adv. Space Res., 58, 453

Palmaerts, B., Roussos, E., Krupp, N., et al. 2016, Icarus, 271, 1

Palmer, I. D. 1982, Rev. Geophys. Space Phys., 20, 335

Pesses, M. E., \& Goertz, C. K. 1976, Geophys. Res. Lett., 3, 228

Potgieter, M. S. 2014, Braz. J. Phys., 44, 581

Potgieter, M. S., \& Nndanganeni, R. R. 2013, Astrophys. Space Sci., 345, 33

Potgieter, M. S., \& Vos, E. E. 2017, A\&A, 601, A23

Potgieter, M. S., Vos, E. E., Munini, R., Boezio, M., \& Di Felice, V. 2015, ApJ, 810,141

Prinsloo, P. L., Potgieter, M. S., \& Strauss, R. D. 2017, ApJ, 836, 100

Pyle, K. R., \& Simpson, J. A. 1977, AJ, 215, L89

Roussos, E., Krupp, N., Mitchell, D. G., et al. 2016, Icarus, 263, 101

Simpson, J. A., Anglin, J. D., Balogh, A., et al. 1992, Science, 257, 1543

Strauss, R. D., Potgieter, M. S., Büsching, I., \& Kopp, A. 2011a, ApJ, 735, 83

Strauss, R. D., Potgieter, M. S., Kopp, A., \& Büsching, I. 2011b, J. Geophys. Res (Space Phys.), 116, A12105

Strauss, R. D., Potgieter, M. S., \& Ferreira, S. E. S. 2013, Adv. Space Res., 51, 339

Teegarden, B. J., McDonald, F. B., Trainor, J. H., Webber, W. R., \& Roelof, E. C. 1974, J. Geophys. Res., 79, 3615

van Allen, J. A., Randall, B. A., Baker, D. N., et al. 1975, Science, 188, 459

Vogt, A., Heber, B., Kuehl, P., Kopp, A., \& Strauss, R. D. 2015, Proc. 34th Int. Cosmic Ray Conf.

Williams, D. J., McEntire, R. W., Jaskulek, S., \& Wilken, B. 1992, Space Sci. Rev., 60, 385

Zhang, M., Qin, G., Rassoul, H., et al. 2007, Planet. Space Sci., 55, 12

Zhang, X.-J., Thorne, R. M., Ma, Q., et al. 2017, Geophys. Res. Lett., 44, 4472 